\title{
Comparação entre os métodos de injeção de toxina botulínica em músculo ocular externo com o uso do eletromiógrafo e com o uso da pinça de Mendonça
}

\author{
Electromyograph assistance and Mendonca's forceps-a comparison between two \\ methods of botulinum toxin A injection into the extraocular muscle
}

\author{
Tomás Fernando Scalamandré Mendonça ${ }^{1}$ \\ Mônica Fialho Cronemberger ${ }^{2}$ \\ Márcia Cordeiro Emery Lopes $^{3}$ \\ Célia Regiina Nakanami ${ }^{4}$ \\ Harley Edson Amaral Bicas ${ }^{5}$
}

\footnotetext{
${ }^{1}$ Médico Oftalmologista do Setor de Motilidade Ocular Extrínseca da Universidade Federal de São Paulo UNIFESP. São Paulo (SP).

Pós-graduanda, do Departamento de Oftalmologia da UNIFESP. São Paulo (SP).

Estagiária do $2^{\circ}$ ano do Setor de Motilidade Ocular Extrínseca da UNIFESP. São Paulo (SP).

${ }^{4}$ Chefe do Setor de Motilidade Ocular Extrínseca do Departamento de Oftalmologia da UNIFESP. São Paulo (SP).

${ }^{5}$ Professor Titular do Departamento de Oftalmologia, Otorrinolaringologia e Cirurgia de Cabeça e Pescoço da Faculdade de Medicina de Ribeirão Preto da Universidade de São Paulo - USP. Ribeirão Preto (SP).

Endereço para correspondência: Rua Botucatu, 824 São Paulo (SP) CEP 04023-062

E-mail: oftalmotsm@aol.com

Recebido para publicação em 01.03.2004

Versão revisada recebida em 17.09.2004

Aprovação em 03.01.2004

O primeiro autor obteve patente da "pinça de Mendonça" usada neste trabalho.
}

\begin{tabular}{|l|}
\hline RESUMO \\
\hline Objetivo: Comparar dois métodos de aplicação de toxina botulínica A \\
(TBA) em músculo ocular externo: com auxílio de eletromiógrafo (EMG) \\
e com a pinça de Mendonça. Métodos: Foram analisados no Departamen- \\
to de Oftalmologia da UNIFESP 29 pacientes que apresentavam estrabis- \\
mo e baixa acuidade visual em um olho. Foram divididos em dois grupos: \\
grupo I - 17 pacientes que receberam a toxina botulínica A por meio de \\
injeção com auxílio da pinça de Mendonça e grupo II - 12 pacientes que \\
receberam a toxina botulínica A por injeção guiada pelo eletromiógrafo. \\
Os pacientes dos dois grupos foram avaliados no 70 e no 140 dia após \\
aplicação. Compararam-se os resultados dos dois grupos neste período \\
de tempo. Os testes de correlação de Friedman e Mann-Whitney foram \\
usados para análise estatística. Resultados: Houve diferença estatística \\
entre as médias de desvio pré-aplicação e em pelo menos um período (70 \\
ou 14ㅇ dia) após aplicação, tanto no grupo dos pacientes em que foi \\
utilizada a pinça, quanto no grupo de pacientes em que foi utilizado o \\
eletromiógrafo. Não houve diferença estatística dos desvios pré-aplica- \\
ção e pós-aplicação entre os dois grupos. Conclusão: Os dois métodos \\
de aplicação da toxina botulínica A são equivalentes e portanto, o uso \\
da pinça de Mendonça pode ser método alternativo ao uso do eletromió- \\
grafo, para guiar a injeção de toxina botulínica A.
\end{tabular}

Descritores: Estrabismo/quimioterapia; Toxina botulínica tipo A/uso terapêutico; Toxina botulínica tipo A/administração \& dosagem; Injeções; Músculos oculomotores: Eletromiografia; Ambliopia; Resultado de tratamento

Objetivo: Comparar dois métodos de aplicação de toxina botulínica A (TBA) em músculo ocular externo: com auxílio de eletromiógrafo (EMG) to de Oftalmologia da UNIFESP 29 pacientes que apresentavam estrabismo e baixa acuidade visual em um olho. Foram divididos em dois grupos: injeção com auxílio da pinça de Mendonça e grupo II - 12 pacientes que receberam a toxina botulínica A por injeção guiada pelo eletromiógrafo.

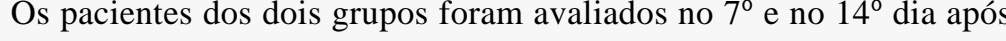
de tempo. Os testes de correlação de Friedman e Mann-Whitney foram entre as médias de desvio pré-aplicação e em pelo menos um período (7ํㅜ ou 14ํ dia) após aplicação, tanto no grupo dos pacientes em que foi utilizada a pinça, quanto no grupo de pacientes em que foi utilizado o ção e pós-aplicação entre os dois grupos. Conclusão: Os dois métodos de aplicação da toxina botulínica A são equivalentes e portanto, o uso da pinça de Mendonça pode ser método alternativo ao uso do eletromiógrafo, para guiar a injeção de toxir

\section{INTRODUÇÃ̃O}

A injeção de toxina botulínica A (TBA) em músculos oculares externos segundo a técnica original (descrita por Scott) baseia-se no uso do eletromiógrafo (EMG) para a localização do músculo alvo ${ }^{(1)}$.

Esta técnica possui algumas desvantagens que limitam o amplo uso da toxina botulínica na prática estrabismológica, como a do alto custo do aparelho, a do uso de agulhas especiais descartáveis além da necessidade do uso do anestésico cetamina, quando da aplicação em crianças.

Em vista disso, alguns autores já utilizam outros métodos para a aplicação da toxina botulínica ${ }^{(2-3)}$. Mendonça em 1999, desenvolveu uma técnica alternativa para este fim, que utiliza uma pinça com desenho apropriado para guiar a injeção da toxina, com a intenção de ser um método mais prático e tão eficiente quanto o método tradicionalmente utilizado (Figura 1). Ape- 


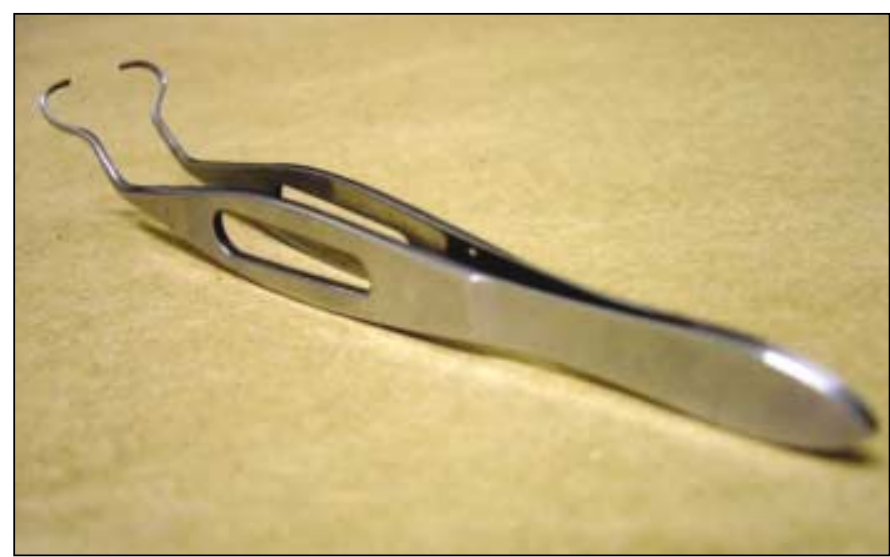

Figura 1 - Pinça de Mendonça

sar de não publicada, ela vem sendo usada em muitos serviços com bons resultados.

A utilização da pinça foi inicialmente idealizada para uso em crianças, pois, o uso de anestesia geral abole o ruído produzido pelo aparelho EMG, mesmo quando a agulha atinge o músculo, tornando inviável o seu uso com simples sedação. A exceção é o uso do anestésico cetamina endovenoso em doses baixas, que por causar nistagmo permite que o sinal emitido pelo EMG seja preservado e com isso o músculo alvo possa ser encontrado. Porém, tal procedimento é contra-indicado por vários anestesistas pelo relato de complicações (alucinações e hipertensão arterial sistêmica) relacionadas com este anestésico.

Portanto, a injeção de TBA em músculo ocular externo em crianças com uso da pinça é muito mais fácil de ser praticada; e embora, ainda não analisados estatisticamente, em nossa experiência os resultados quanto à eficiência imediata são muito próximos quando comparados com o EMG.

A literatura revela que os melhores resultados do uso da TBA em adultos foram obtidos em pacientes com hipo e hipercorreções cirúrgicas, paralisias recentes de VI nervo e esotropias de pequeno ângulo ${ }^{(1,4-5)}$. No entanto, outros autores, mostraram a utilização dessa droga em pacientes com exotropia de grande ângulo e baixa acuidade visual em um olho na tentativa de diminuir o número de músculos oculares externos operados $^{(6)}$. Outros trabalhos já citaram, também, o uso da toxina em desvio de grande ângulo ${ }^{(7)}$.

Este trabalho compara resultados entre este novo método e o tradicional, guiado por eletromiógrafo, em pacientes adultos com desvios de grande ângulo e baixa de visão unilateral.

\section{MÉTODOS}

Foram estudados 29 pacientes atendidos no Setor de Estrabismo do Departamento de Oftalmologia na EPM-UNIFESP, que apresentavam desvio de grande ângulo $\left(\geq\right.$ a $\left.40^{\Delta}\right)$, com baixa visão em um dos olhos.

Todos os pacientes foram submetidos a exame oftalmoló- gico antes e após aplicação da toxina, que constou de medida da acuidade visual, exame de motilidade ocular (medida do desvio, versões e duções), mapeamento da retina e refração.

Pacientes com cirurgia oftalmológica prévia foram excluídos.

$\mathrm{O}$ ângulo de desvio foi medido com prismas pelo teste de cobertura simples; ou com o método de Krimsky quando houve impossibilidade de fixação.

Dos 29 indivíduos estudados, com idade variando entre 14 anos e 66 anos, 20 pacientes tinham esotropia (ET) e 9 exotropia (XT). Doze receberam a toxina guiada por eletromiógrafo ( 7 homens e 5 mulheres) e 17 receberam a toxina guiada pela pinça (5 homens e 12 mulheres).

Todos os pacientes apresentaram acuidade visual abaixo de 0,4 em pelo menos um olho. Dentre as causas de baixa acuidade visual foram encontradas: ambliopia estrabísmica em 21 pacientes, placa macular em um paciente, atrofia de nervo óptico primária em dois pacientes, leucoma corneano (um paciente), descolamento de retina (um paciente), miopia degenerativa (um paciente), coloboma de retina (um paciente). Após aprovação do comitê de ética da UNIFESP, os pacientes foram sorteados para receber a aplicação da toxina botulínica guiada pelo eletromiógrafo ou pela pinça.

Nos pacientes, independente do método a ser escolhido, a anestesia foi sempre tópica (Cloridrato de proximetacaína $0,5 \%$ ). Foram instiladas pelo menos três gotas desse colírio antes do procedimento.

A toxina botulínica tipo A utilizada neste estudo foi do laboratório Allergan $\left(\right.$ Botox $\left.^{\circledR}\right)$. O conteúdo liofilizado do frascoampola foi diluído em $2 \mathrm{ml}$ de solução salina sem conservante.

A técnica utilizada para a aplicação de toxina botulínica nos músculos oculares externos (MOE) com ajuda de eletromiógrafo (EMG) foi a tradicionalmente descrita por $\mathrm{Scott}^{(1)}$. Esta utiliza uma agulha especial descartável revestida por silicone; só a sua ponta fica livre, funcionando como um eletrodo monopolar acoplado ao EMG que emite um sinal sonoro, para assegurar a colocação da agulha no músculo. Tanto a agulha quanto o EMG usados neste estudo são fabricados pela Allergan.

A técnica alternativa proposta por um dos autores (T.F.S.M.) é realizada da seguinte forma: após colocação de blefarostato, uma pinça de conjuntiva apreende a conjuntiva na região limbar próxima ao músculo alvo e é então realizada uma dução passiva em sentido oposto ao da ação principal daquele. A pinça "de Mendonça" (Figura 1) é posicionada aberta e pressionada contra a esclera na região onde supostamente se localiza o músculo, posteriormente à sua inserção. Ao fechar a pinça, o corpo muscular fica apreendido no interior do anel. Para certificar-se que o músculo esteja contido neste anel, desloca-se a pinça em direção ao limbo e esta deverá parar na altura da inserção muscular. Em seguida é feita a injeção de toxina botulínica tipo A com auxílio de agulha comum de insulina, tamanho $27 \mathrm{G}$.

A dose da toxina injetada com qualquer dos métodos foi calculada de acordo com a magnitude do desvio ocular, segundo o seguinte esquema: 5 unidades para desvio menores que $50^{\Delta}$ e 7,5 unidades para os de magnitude igual ou superior a $50^{\Delta}$. 
Os pacientes foram reavaliados no $7^{0}$ e $14^{0}$ dia e comparouse o desvio pré-existente com os desvios obtidos nas duas aferições. Foram comparados os resultados dos dois métodos neste período de tempo.

Para avaliar possíveis diferenças entre os períodos de tempo para a variável medida do desvio em dioptrias prismáticas, em cada grupo (pinça ou eletromiógrafo) usou-se o teste não paramétrico para " $k$ " amostras não independentes de Fried$\operatorname{man}^{(8)}$, complementado quando necessário pelo teste de comparações múltiplas ${ }^{(9)}$.

Para comparar possíveis diferenças entre os grupos tanto no $7^{0}$ dia quanto no $14^{\circ}$ dia para a mesma variável usou-se o teste não paramétrico para duas amostras independentes de Mann-Whitney ${ }^{(8)}$. Nesta comparação preferiu-se medir o percentual de variação do período em causa ( $7^{0}$ dia ou $14^{\circ}$ dia) em relação ao período pré-injeção, considerando-se evidentemente o valor pré como sendo $100 \%$. Usou-se esta variável transformada, pois, valores iguais no mesmo período de tempo podem ter significado diferente quando observado o valor pré.

O nível de rejeição para a hipótese de nulidade foi fixado sempre em um valor menor ou igual do que $0,05(5 \%)$. Quando a estatística calculada apresentou significância usou-se um asterisco (*) para caracterizá-la. Caso contrário, isto é, não significante usou-se N.S.

As médias dos desvios (consideradas sem seus sinais) foram calculadas e apresentadas a título de informação.

Não se calcularam os desvios-padrão, pois, usando-se testes não paramétricos estamos pressupondo que a variável em causa não se comporta como curva de Gauss e, portanto, não há sentido em seu cálculo.

\section{RESULTADOS}

As medidas pré e pós-aplicação estão nas tabelas I e II. A média de correção do grupo do eletromiógrafo foi 44,3D
$\left(68^{\Delta}\right.$ a $\left.20^{\Delta}\right)$ no $7^{0}$ dia e $40,0^{\Delta}\left(65^{\Delta}\right.$ a $\left.10^{\Delta}\right)$ no $14^{\circ}$ dia. Dos pacientes do grupo Pinça a média de correção foi de $35,9^{\Delta}\left(70^{\Delta}\right.$ a 0$)$ no $7^{0}$ dia e $41,8^{\Delta}\left(90^{\Delta}\right.$ a 0$)$ no $14^{\circ}$ dia.

As tabelas III e IV apresentam as médias de correção nos períodos de tempo dos métodos EMG e pinça, respectivamente, e a tabela V e VI apresentam as comparações dos dois métodos EMG e Pinça nos 7으 e 14을 dias, respectivamente, com valores estatísticos. Para o estudo estatístico foram considerados apenas os pacientes que compareceram no sétimo e no $14^{0}$ dia (no grupo do EMG foram 7 pacientes e no grupo da Pinça foram 16 pacientes). Foram excluídos aqueles que por algum motivo deixaram de comparecer em pelo menos um dos retornos avaliados.

Para análise da tabela $\mathrm{V}$ foram considerados os pacientes que retornaram no $7^{0}$ dia e para análise da tabela VI foram considerados os pacientes que retornaram no $14^{\circ}$ dia.

As complicações observadas em ambos grupos foram blefaroptose e desvio vertical. Não houve casos de perfuração ocular ou alterações sistêmicas. As complicações serão objetos de estudo em outro trabalho.

\section{DISCUSSÃO}

Antes de comparar os resultados é interessante lembrar que o método tradicional de aplicação de TBA depende de um aparelho de eletromiografia, sofisticado e caro. O mais comumente usado para este fim é o EMG fornecido pela Allergan com localização do músculo por sinal sonoro e só é útil para esta finalidade, o que torna sua aquisição do ponto de vista financeiro não compensadora.

Além disso, a curva de aprendizado para correta aplicação da técnica não é das mais rápidas. No presente estudo a série de pacientes que fizeram uso do EMG não é inicial, ou seja, já havia larga experiência no seu uso em outros pacientes no nosso serviço.

Em adultos a experiência clínica do uso da pinça ainda é

\begin{tabular}{|cccccc|}
\hline \multicolumn{7}{|c|}{ Tabela I. Medidas do desvio pré-aplicação e no 70 e 140 dia pós-aplicação de toxina botulínica em pacientes que utilizaram o método guiado } \\
por eletromiógrafo \\
Paciente
\end{tabular}




\begin{tabular}{|c|c|c|c|c|c|c|}
\hline \multicolumn{7}{|c|}{ Pinça } \\
\hline Paciente & Músculo realizado & Desvio-pré & Desvio 1a semana & \% correção & Desvio 2a semana & \% correção \\
\hline $1 \mathrm{P}$ & RME & ET $70^{\Delta}$ & Orto & 100,0 & Orto & 100,0 \\
\hline $2 \mathrm{P}$ & RMD & ET50 ${ }^{\Delta}$ HTD15 $^{\Delta}$ & HTD10 ${ }^{\Delta}$ & 100,0 & $\mathrm{HTD}^{\Delta}{ }^{\Delta}$ & 100,0 \\
\hline $3 P$ & RME & $\mathrm{ET}^{4} 0^{\Delta} \mathrm{HTD}^{2} 2^{\Delta}$ & $\mathrm{ET}^{2} 0^{\Delta} \mathrm{HTD}^{2} 2^{\Delta}$ & 0 & $\mathrm{ET} 40^{\Delta} \mathrm{HTD}^{2} 2^{\Delta}$ & 0 \\
\hline $4 \mathrm{P}$ & RME & ET80 $0^{\Delta}$ & ET20 $0^{\Delta} \mathrm{HTE} 10^{\Delta}$ & 75,0 & $\mathrm{ET}^{2} 5^{\Delta} \mathrm{HTE}^{\Delta}{ }^{\Delta}$ & 68,8 \\
\hline $5 P$ & RMD & $\mathrm{ET}^{\mathrm{E}} 0^{\Delta}$ & ET30 $0^{\Delta}$ & 57,1 & ET30 ${ }^{\Delta}$ & 57,1 \\
\hline $6 \mathrm{P}$ & RME & ET80 & ET35 ${ }^{\Delta}$ & 56,3 & ET20 & 75,0 \\
\hline $7 P$ & RME & $\mathrm{ET}^{\circ} 0^{\Delta}$ & ET30 & 50,0 & $\mathrm{ET}_{20} 0^{4}$ & 66,7 \\
\hline $8 \mathrm{P}$ & RMD & $\mathrm{ET70}^{\Delta}$ & ET30 & 57,1 & $\mathrm{XT20^{ \Delta }}$ & 128,6 \\
\hline $9 \mathrm{P}$ & RMD & ET50 ${ }^{\Delta}$ & $\mathrm{XT6}^{\Delta}$ & 112,0 & $\mathrm{XT}^{\Delta}{ }^{\Delta}$ & 108,0 \\
\hline $10 \mathrm{P}$ & RME & ET90 ${ }^{\Delta}$ HoTE $15^{\Delta}$ & $\mathrm{ET}_{4} 0^{\Delta} \mathrm{HoTE}^{\Delta}$ & 55,6 & ET35 $^{\Delta}$ HoTE $^{\Delta}$ & 61,1 \\
\hline $11 \mathrm{P}$ & RME & ET70 ${ }^{\Delta}$ & ET15 $^{\Delta}$ HTE $^{\Delta}{ }^{\Delta}$ & 78,6 & ET10 $^{\Delta}$ HTE $^{\Delta}$ & 85,7 \\
\hline $12 \mathrm{P}$ & RLD & $\mathrm{XT75^{ \Delta }}$ & XT55 ${ }^{\Delta} \mathrm{HTD6}^{\Delta}$ & 26,7 & XT55 & 26,7 \\
\hline $13 P$ & RLD & XT55 $5^{\Delta}$ & $\mathrm{XT25^{ \Delta }}$ & 54,5 & & \\
\hline $14 \mathrm{P}$ & RLD & $\mathrm{XT} 70^{\Delta}$ & $\mathrm{XT} 40^{4}$ & 42,9 & XT50 & 28,6 \\
\hline $15 \mathrm{P}$ & RLD & $\mathrm{XT} 65^{\Delta}$ & $\mathrm{XT} 60^{\Delta}$ & 7,7 & $\mathrm{XT50^{ \Delta }}$ & 23,1 \\
\hline $16 \mathrm{P}$ & RLD & $\mathrm{XT} 60^{\Delta}$ & XT30 & 50,0 & XT20 ${ }^{\Delta}{\text { HoTD} 10^{\Delta}}^{\Delta}$ & 66,7 \\
\hline $17 \mathrm{P}$ & RLD & $\mathrm{XT90^{ \perp }}$ & $\mathrm{XT} 90^{\Delta}$ & 0 & XT90 & 0 \\
\hline
\end{tabular}

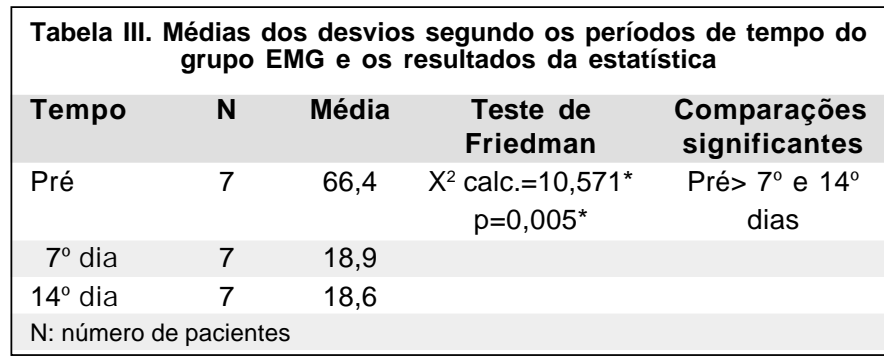

\begin{tabular}{|c|c|c|c|c|}
\hline \multicolumn{5}{|c|}{$\begin{array}{c}\text { Tabela IV. Médias dos desvios segundo os períodos de tempo do } \\
\text { grupo pinça e resultados estatísticos }\end{array}$} \\
\hline Tempo & $\mathbf{N}$ & Média & $\begin{array}{l}\text { Teste de } \\
\text { Friedman }\end{array}$ & $\begin{array}{l}\text { Comparações } \\
\text { significantes }\end{array}$ \\
\hline Pré & 16 & 68,1 & $\begin{array}{l}X^{2} \text { calc. }=23,231^{*} \\
\quad p=0,000^{*}\end{array}$ & $\begin{array}{l}\text { Pré> } 70 \text { e } 14^{0} \\
\quad \text { dias }\end{array}$ \\
\hline $7^{0} \mathrm{dia}$ & 16 & 31,8 & & \\
\hline $14^{0} \mathrm{dia}$ & 16 & 26,3 & & \\
\hline
\end{tabular}

pequena, mas os resultados apresentados mostram que é tão eficiente quanto o método tradicional. As complicações presentes foram as mesmas nos dois grupos.

Comparando os resultados dos dois grupos (EMG e Pinça) observou-se que:

a) Os resultados da tabela III (EMG) mostram que o valor da estatística calculada $\left(\mathrm{X}^{2}\right.$ calc. $\left.=10,571^{*}\right)$ revela que há diferença estatisticamente significante $\left(\mathrm{p}=0,005^{*}\right)$ em pelo menos um período e o teste de comparações múltiplas mostrou que o período pré $(66,4)$ foi significantemente maior que os períodos

\begin{tabular}{|lccc|}
\hline \multicolumn{4}{|c|}{$\begin{array}{c}\text { Tabela V. Médias das percentagens de correção dos desvios entre } \\
\text { os grupos no 70 dia e o resultado da estatística }\end{array}$} \\
Grupo & N & Média & Teste de Mann-Whitney \\
EMG & 10 & 69,9 & Zcalc. $=-1,307$ N.S. \\
Pinça & 17 & 54,3 & $P=0,191$ N.S. \\
N: número de pacientes; EMG: Eletromiógrafo; N.S.: não significante \\
\hline
\end{tabular}

\begin{tabular}{|l|ccc|}
\hline \multicolumn{4}{|c|}{$\begin{array}{c}\text { Tabela VI. Médias das percentagens de correção dos desvios entre } \\
\text { os grupos no }\end{array}$} \\
140 dia e o resultado da estatística \\
Grupo & N & Média & Teste de Mann-Whitney \\
EMG & 9 & 64,9 & Zcalc. $=-0,255$ N.S. \\
Pinça & 16 & 62,2 & P=0,799 N.S. \\
N: número de pacientes; EMG: Eletromiógrafo; N.S.: não significante \\
\hline
\end{tabular}

do $7^{0}$ dia $(18,9)$ e do $14^{\circ}$ dia $(18,6)$. Não houve diferença significante entre o $7^{0}$ e $14^{\circ}$ dias.

b) Os resultados da tabela IV (Pinça), mostram que o valor da estatística calculada $\left(\mathrm{X}^{2}\right.$ calc $\left.=23,231 *\right)$ revela os mesmos resultados que a tabela anterior, ou seja, há apenas diferença estatisticamente significante quando se compara o período pré $(68,1)$ com os períodos $7^{0}(31,8)$ e $14^{\circ}(26,3)$ dias.

c) A tabela $\mathrm{V}$ mostra que no $7^{\circ}$ dia o percentual de melhora no grupo EMG foi igual a $69,9 \%$ enquanto que na Pinça o porcentual foi igual a 54,3\%; o teste de Mann-Whitney apresentou um valor de Zcalc. $=-1,307$ N.S ( $p=0,191 \%$ N.S). Notase, portanto, que não houve diferença estatisticamente significante entre os grupos.

d) A tabela VI mostra igualmente que não houve diferença estatisticamente significante Zcal.=-0,255 N.S. $(p=0,799$ N.S.) 
entre o porcentual de melhora entre os grupos da pinça e EMG, no $14^{\circ}$ dia.

Estes dados mostram que no $7^{\circ}$ dia a melhora no grupo EMG foi praticamente igual à do $14^{\circ}$ dia e, portanto, a melhora é brusca no $7^{\circ}$ dia em relação ao pré, porém, se mantém no $14^{\circ}$ dia. No grupo da pinça a melhora no $7^{0}$ dia não se apresentou tão acentuada quanto no grupo do EMG, porém no 14ํessa melhora continuou ocorrendo em relação ao 7ํㅡㄹia, verificando-se, portanto, que a melhora no grupo da pinça é constante e gradativa. Esta diferença não era esperada, e a nossa hipótese para tal é que a injeção pelo EMG por utilizar agulha um pouco mais longa que a agulha de insulina, possivelmente coloca a TBA mais próxima da junção mio-neural fazendo seu efeito aparecer mais rápido, embora o resultado final seja o mesmo.

\section{CONCLUSÕES}

1) Os dois métodos são equivalentes.

2) O efeito do eletromiógrafo aparece um pouco mais cedo do que o da pinça, porém, após 15 dias eles passam a ser praticamente iguais.

\section{ABSTRACT}

Purpose: To compare two methods of botulinum toxin A (BTA) injection into the extraocular muscle (EOM): the electromyographically (EMG) guided injection and the injection using Mendonça's forceps. Methods: Twenty-nine (29) patients with strabismus and low visual acuity in one eye were examined at the Department of Ophthalmology of UNIFESP. They were divided into 2 groups - group I with 17 patients receiving the botulinum toxin A injection using Mendonça's forceps, and group II with 12 patients receiving the toxin with electromyographical guidance. The patients of both groups were examined on the $7^{\text {th }}$ and $14^{\text {th }}$ day after intervention and the outcome of both methods of botulinum toxin A injection were compared. Friedman and Mann-Whitney correlation tests were used in the statistical analysis of the data. Results: Although the follow-up examinations on the $7^{\text {th }}$ and $14^{\text {th }}$ days showed a different behavior between both methods, there was no statistically significant difference between the mean values of the correction attained in both groups at the end of this study. Conclusion: No statistically significant difference was shown between the two groups. Mendonça's forceps can be a safe alternative to electromyography to locate an extraocular muscle for botulinum toxin A injection.

Keywords: Strabismus/drug therapy; Botulinum toxin type A/ therapeutic use; Botulinum toxin type A/administration \& dosage; Injections; Oculomotor muscles; Electromyography; Amblyopia; Treatment outcome

\section{REFERÊNCIAS}

1. Scott AB. Botulinum toxin injection of eye muscles to correct strabismus. Trans Am Ophth Soc. 1981;79:734-70.

2. Benabent EC, Garcia Hermosa P, Arrazola MT, Alió Sanz JL. Botulinum toxin injection without electromyographic assistance. J Pediatr Ophthalmol Strabismus. 2002;39(4):231-4

3. Kao LY, Chao AN. Subtenon injection of botulinum toxin for treatment of traumatic sixth nerve palsy. J Pediatr Ophthalmol Strabismus. 2003;40(1):27-30.

4. Biglan AW, Burnstine RA, Rogers GL, Saunders RA. Management of strabismus with botulinum A toxin. Ophthalmology. 1989;96(7):935-43.

5. Osako M, Keltner JL. Botulinum A toxin (oculinum) in ophthalmology. Surv Ophthalmol. 1991;36(1):28-46.

6. Owens PL, Strominger MB, Rubin PA, Veronneau-Trouman S. Large-angle exotropia corrected by intraoperative botulinum toxin A and monocular recession resection surgery. J AAPOS 1998:2(3):144-6.

7. Elston JS, Lee JP, Powell CM, Hogg C, Clark P. Treatment of strabismus in adults with botulinum toxin A. Br J Ophthalmol. 1985;69(10):718-24.

8. Siegel S. Estatística não-paramétrica (para as ciências do comportamento). São Paulo: Mc Graw-Hill; 1975. 350p.

9. Holander M, Wolf DA. Nonparametric statistical methods. New York: John Wiley e Sons; 1973. 503p.

\section{SIMPÓSIO DA SOCIEDADE BRASILEIRA DE GLAUCOMA}

$$
19 \text { a } 21 \text { de maio de } 2005
$$

Frei Caneca Shopping \& Convention Center SÃO PAULO - SP

\section{IN FO RMAÇÕES: JDE Comunicação e Eventos}

Tels.: (11) 5082-3030 / 5084-5284

Fax: (11) 5574-8261 - E-mail: jdecomev@uol.com.br 